\title{
Emerging Blood Culture Technologies for Isolation of Blood Pathogens at Clinical Microbiology Laboratories
}

\author{
Tebelay Dilnessa $^{1^{*}}$, Gebresilasie Demeke ${ }^{1}$ Getachew Mengistu ${ }^{1}$ and Adane Bitew ${ }^{2}$ \\ ${ }^{1}$ Department of Medical Laboratory Sciences, College of Health Sciences, Debre Markos University, Debre Markos, Ethiopia \\ ${ }^{2}$ Department of Medical Laboratory Sciences, College of Health Sciences, Addis Ababa University, Addis Ababa, Ethiopia
}

"Corressponding author: Dilnessa T, Department of Medical Laboratory Sciences, College of Health Sciences, Debre Markos University, Debre Markos, Ethiopia, Tel: 251912198715; E-mail: tebelay@gmail.com

Rec Date: Mar 03 2016; Acc Date: Apr 24 2016; Pub Date: Apr 282016

Copyright: $\odot 2016$ Dilnessa T, et al. This is an open-access article distributed under the terms of the Creative Commons Attribution License, which permits unrestricted use, distribution, and reproduction in any medium, provided the original author and source are credited.

\begin{abstract}
Bloodstream infections (BSIs) are important cause of morbidity and mortality in patients. Around 20 million cases of severe sepsis arise each year worldwide. Rapid and accurate identification of the etiologic pathogen is crucial to be able to initiate pathogen specific antibiotic therapy and decrease mortality rates, and for reducing length of hospitalization and hospital costs. These identification techniques are applied on various emerging blood culture techniques. This review summarized and presented the currently available technologies and different strategies of conventional laboratory diagnostic steps for blood stream infection. Based on their capability these techniques can be manual, semi-automated or fully automated. Considering the importance of blood cultures in diagnosis and management of bloodstream infections, it is prudent and relevant to understand the principles, technical requirements and limitations of these technologies.
\end{abstract}

Nowadays, there are various emerging blood culture techniques for the diagnosis of blood stream infections and drug susceptibility testing. Among these are API, the BBL systems, the BACTEC systems, the VITEK systems, the BacT/Alert, the BacT/Alert 3D and the Versa TREK system can be mentioned. These systems depend on various detection principles and cultural environments to detect microorganisms. The selection of instrument for the identification and susceptibility testing of bacteria in diagnostic laboratory rely on speed, accuracy, ease of use and cost factors. All marketed identification systems are based on one of five different technologies or a combination of them. These include $\mathrm{pH}$-based reactions, enzyme-based reactions, utilization of carbon sources, visual detection of bacterial growth, or detection of volatility fatty acids.

Keywords: Blood culture; Bacteria pathogen; Emerging blood culture techniques

\section{Introduction}

Bloodstream infections are important cause of morbidity and mortality in patients $[1,2]$. About 20 million cases of severe sepsis arise every year worldwide. In the USA, septicemia was consistently occurred in the top 10 causes of death, accounting for 35,587 deaths in 2009 alone. In an Australia hospital, mortality for patients presenting with septic shock ranges from $23.1-27.6 \%$. Blood culture systems and practices that maximize the recovery of pathogens are imperative in the investigation of septicemic patients to ensure the timely delivery of appropriate antimicrobials [3,4]. Blood culture is clearly the most important diagnostic procedure for identifying microorganisms involved in bloodstream infections. Ideally, blood samples should be taken immediately prior to the start of empirical antimicrobial treatment. However, the blood culture is slow and insufficiently sensitive when the patient has previously received antibiotics or in the presence of slow-growing or intracellular microorganisms [2]. The strains that display $a$ and $\beta$-hemolysis have exotoxins that may display harmful effects within body as evidenced by their breakdown of erythrocytes in blood agar medium [4].

Bacteriological analysis of blood samples is routinely carried out by selective culturing of blood which has been incubated in a blood culture system. Currently, three continuously reading, automated blood culture systems are available in clinical microbiology: the BACTEC 9240, the BacT/ALERT and the Extra Sensing Power systems. All these machines measure the rate of production of bacterium-specific metabolite in the culture bottle containing the patient's blood supplemented with general nutrient broth. Subsequently, if bacterial metabolic activity is detected, the positive blood culture sample is plated on an appropriate selective medium for further analysis. When the positive blood culture is taken as a starting point, microbial identification takes 24-72 hrs to complete [5].

Multiple blood culture techniques can be used for the identification of bacteria from blood specimens. These include direct inoculation of blood-broth aspirates onto Vitek test cards, chromogenic or fluorogenic substrates, enzymic test strips and commercial biochemical identification kits. Molecular testing methods have utilized fluorescent hybridization probes and amplification of 16 SrRNA genes from positive blood culture vials [6]. Automated blood culture systems are commonly used to identify infectious agents causing septicaemia in human medicine. The BACTEC 9240 system is one of the new automated continuous blood culture systems using infrared spectrophotometry to monitor $\mathrm{CO}_{2}$ production by growing bacteria with a fluorescence sensor. This system detects blood-borne organisms rapidly and accurately. It decreases the incubation time, contamination risk and false negative rate [7]. 


\section{Blood Sampling for Culture}

Blood culture is a standard technique for the diagnosis of bloodstream infections (BSIs). It ranges from in house and commercial manual systems to fully automated commercial systems. It establishes the infectious etiology of patient's illness and provides organisms for antimicrobial susceptibility testing and antimicrobial therapy. Regarding the importance of blood cultures in the diagnosis and management of sepsis, it is prudent and relevant to understand the clinical and technical requirements and limitations of this technology [8]. The important factors in the ability of blood cultures to detect significant organisms include:

- Volume of blood drawn (greater or equal to10 $\mathrm{ml}$ of blood for adults and 1-5 ml for infants).

- Dilution: the ratio of blood to culture medium in the blood culture bottle.

- Number of cultures taken (two or more specimens collected).

- Blood culture technique, including skin preparation, obtaining blood via veni puncture rather than from an intravascular catheter and choice of culture site.

- Timing of culture (time of taking blood culturing).

- Choice of blood culture bottle and system (including whether it preferentially detects aerobic or anaerobic organisms) $[9,10]$.

\section{Emerging Blood Culture Techniques}

Nowadays, there are various emerging blood culture techniques for the diagnosis of blood stream infections and drug susceptibility testing. These involve starting from manual methods, semi-automated and more sophisticated fully automated methods. Among these are API, BBL systems, BACTEC systems, VITEK systems, BacT/Alert, BacT/ Alert 3D, Versa TREK system, etc. These systems rely on a variety of detection principles and cultural environments to detect microorganisms. Many systems and their respective media have been compared, each system having its own limitations and advantages. Fully automated continuous monitoring systems are simple to use in comparison with manual and semi-automated systems $[8,11]$.

The selection of automated equipment for the identification and susceptibility testing of bacteria in a diagnostic laboratory depends on speed, accuracy, ease of use, and cost factors [12]. All commercial identification systems are based on one of five different technologies or a combination of them. These include $\mathrm{pH}$-based reactions that require from 15-24 hrs of incubation, enzyme-based reactions that require 2-4 hrs, utilization of carbon sources, visual detection of bacterial growth, or detection of volatile or non-volatile fatty acids via gas chromatography. In $\mathrm{pH}$-based reactions, a positive test is indicated by a change in the color of one or more dyes. When a carbohydrate is utilized, the $\mathrm{pH}$ becomes acidic; when protein is utilized, there is release of nitrogen-containing compounds or the $\mathrm{pH}$ becomes alkaline. These reactions are influenced by the inoculums size, incubation time and temperature of the reaction [13].

\section{Biochemical identification methods}

The API identification systems consist of series of micro cupules on a plastic strip that contain dehydrated substrates for the demonstration of enzymatic activity or the fermentation of carbohydrates. Depending on type of the organism and the API strip utilized, it may or may not require microbial growth. API systems are manual and do not incorporate antimicrobial susceptibility testing [14]. The biochemical identification system tests are based on the biological activities of bacteria in using different substrates and release a certain product. The most commonly known biochemical tests are the BBL Crystal identification system, API identification systems and BBL Phoenix identification and Susceptibility System.

BBL crystal identification system: BBL Crystal system is a miniaturized bacterial identification method employing modified conventional and chromogenic substrates. There are two products currently available: the Rapid Stool/Enteric ID Kit (RS/E kit) and the Enteric/Nonfermenter ID Kit (E/NF kit). Each is based on modified conventional and chromogenic substrates contained within a novel plate. Each kit comprises thirty tests, a plastic base containing reaction wells to which, following inoculation, is clipped a lid with dehydrated substrates on the tips of plastic prongs $[14,15]$.

BBL crystal enteric/non-fermenter system: The BBL Crystal Enteric/ Nonfermenter System was used to test 25 archived isolates of Yersinia pestis to obtain a unique biochemical profile code for $Y$. pestis. Twenty-five isolates of $Y$. pestis from the archived collection at the Centers for Disease Control and Prevention were chosen to represent geographical and host diversity. The biochemical diversity of these isolates was determined with the API 20E system [16].

BBL crystal anaerobe (ANR): BBL crystal anaerobe identification system is a miniaturized $4 \mathrm{hrs}$ identification method employing modified conventional, fluorogenic, and chromogenic substrates to identify anaerobic bacteria from clinical specimens without the need for anaerobic incubation. The BBL Crystal ANR ID kit consists of 20 panel lids, 20 bases and 20 tubes of inoculum fluid. Many of the tests used in this system are modifications of classical methods and include tests for fermentation, oxidation, and degradation or hydrolysis of various substrates. In addition, this system uses chromogenic and fluorochrome-linked substrates to detect preformed metabolic enzymes. The system correctly identified all tested strains of Campylobacter, Desulfomonas, Leptotrichia, Mobiluncus, Peptostreptococcus, Porphyromonas [14,17].

BBL crystal neisseria/haemophilus: The BBL crystal neisseria/ haemophilus $(\mathrm{N} / \mathrm{H})$ identification system is a miniaturized $4 \mathrm{hrs}$ identification method employing modified conventional, fluorogenic, and chromogenic substrates. It is important for the identification of Neisseria, Haemophilus, Moraxella, Gardnerella vaginalis, as well as other fastidious bacteria $[14,18]$.

\section{BBL phoenix identification and susceptibility system}

The BBL phoenix is an automated identification and susceptibility system that can identify clinically significant Gram +ve/-ve microorganisms. The Phoenix ID panel utilizes a series of conventional, chromogenic, and fluorogenic biochemical tests to determine the identification of the organism. Both growth-based and enzymatic substrates are employed to cover the different types of reactivity. The tests are based on microbial utilization and degradation of specific substrates detected by various indicator systems. Acid production is indicated by a change in phenol red indicator when an isolate is able to utilize a carbohydrate substrate. Chromogenic substrates produce a yellow color upon enzymatic hydrolysis of either p-nitrophenyl or p-nitroanilide compounds. Enzymatic hydrolysis of fluorogenic substrates results in the release of a fluorescent coumarin derivative. Organisms that utilize a specific carbon source reduce the resazurin-based indicator [14]. 


\section{API identification systems}

API 20E: The API 20E test was first evaluated by Washington in 1971, and originally owned by the Analytab products division of American Home Products, which has been owned since 1986. An impermeable plastic backing supports 20 cupules that contain $\mathrm{pH}$ based substrates [13]. It is an identification system for Enterobacteriaceae and other non-fastidious of non-fermenting Gramnegative rods, which uses 21 standardized and miniaturized biochemical tests and a database. It consists of 21 micro-tubes containing dehydrated substrates. These tubes are inoculated with a bacterial suspension which reconstitutes the media. During incubation, metabolism produces color changes that are either spontaneous or revealed by the addition of reagents. The reactions are read according to the table provided and the identification is done using the software provided by the manufacturer on API web $[19,20]$.

API 20NE: API 20NE is constructed along the same lines as the API $20 \mathrm{E}$ and has 20 cupules that contain 8 conventional substrates and 12 assimilation tests. Suspensions are prepared in $0.85 \% \mathrm{NaCl}$ for inoculation into the 8 conventional substrates and in AUX medium for inoculation into the 12 assimilation cupules. The database contains 32 genera and 64 species of non-fastidious gram-negative rods not belonging to the Enterobacteriaceae. These strains included species of Pseudomonas, Acinetobacter, Achromobacter, Bordetella, Flavobacterium, Alcaligenes and Moraxella [13].

API RapiD 20E: Originally marketed in 1982 as the Rapid E system (DMS Laboratories, Flemington) but owned since 1986 by API and subsequently by bioMe'rieux Inc. The API RapiD $20 \mathrm{E}$ system is designed to identify Enterobacteriaceae in $4 \mathrm{hrs}$. Similar to the API $20 \mathrm{E}$ in its test configuration, this system has 20 microtubes that contain substrates for the demonstration of enzymatic activity or fermentation of carbohydrates. The seven digit profile number that is compiled from the test reactions is entered into the APILAB software [13].

RapID NF Plus: The RapID NF Plus was originally designed in 1989 and marketed by innovative diagnostic systems (IDS), but is now marketed by Remel. It employs both conventional and chromogenic substrates for the identification of non-glucose fermenting, gramnegative bacilli and selected glucose-fermenting bacilli not belonging to the family Enterobacteriaceae. The plastic panel has molded into 10 reaction cavities that contain seventeen substrates. The addition of reagents allows seven of the cavities to be bi-functional, containing two separate tests in the same cavity $[13,19]$.

API NH strip: API NH strip is used for identification of Haemophilus and Neisseria species, based on colorimetric changes in miniaturized substrates, with either enzymatic or growth end points. The ability to identify and separate Haemophilus species from nonpathogenic Neisseria species, particularly those from the respiratory tract, then led to the addition of Haemophilus identification in these systems. BioMe'rieux has developed a new Neisseria-Haemophilus (NH) identification card for the Vitek 2 system. The $\mathrm{NH}$ card is based on colorimetric technology utilizing dehydrated media containing chromogenic substrates [18].

RAPIDEC Staph: The RAPIDEC Staph system is a biochemical test that is used for identification of the principal staphylococcal species, $S$. aureus, $S$. epidermidis, and $S$. saprophyticus. The test includes control and test cupules for fluorogenic detection of coagulase and chromogenic substrates for alkaline phosphatase and betagalactosidase to identify $S$. aureus, $S$. epidermidis, and $S$. saprophyticus, respectively. Positive results with both chromogenic substrates provide a presumptive identification of $S$. xylosus or $S$. intermedius. Test cupules are inoculated with an organism suspension and reactions are read after 2 hrs incubation [21,22].

API 20 Strep: The API 20 Strep is a test used for identification of Streptococcus species. A suspension was made from the culture grown on sheep blood agar plate by transferring a heavy inoculum of the culture to a tube containing $2 \mathrm{ml}$ of distilled water. Three drops of suspension were added into each microcapsule by using a Pasteur pipette. Strips were incubated at $37^{\circ} \mathrm{C}$ in a normal atmosphere. After 4 hrs of incubation, reagents were added and strips were exposed to a strong light for reading enzymatic activities. Test results were then recorded, and the strips were incubated again for 20 hours. The interpretation of test results obtained at 4 and $24 \mathrm{hrs}$ of incubation for identification of Streptococcus species was based on the profile index and table [23].

\section{The BACTEC system}

The BACTEC system measures $\mathrm{CO}_{2}$ important functions of a microbiology laboratory produced by the organisms as they metabolize the blood culture remains central to this quest for identifying utilization of substrates present in the vial [24]. In spite of the vial sensor caused by the higher amount of $\mathrm{CO}_{2}$ is recent developments, such as nucleic acid probes, monitored by the BACTEC fluorescent series instruments. BD has a long history of blood culture technology beginning with the radiometric systems (BACTEC 460) in the early 1970s and followed in the 1980s by the non-radiometric infrared 660 and 730 systems. In 1992 the non-invasive BACTEC 9000 series systems were introduced with a change in the technology to a fluorescent indicator which detects increases the levels of carbon dioxide emitted by an organism. Blood cultures are incubated in $\mathrm{CO}_{2}$ incubator $[24,25]$.

BACTEC 460 TB: The most commonly used conventional Lowenstein Jensen (L.J) culture method needs at least 6-10 weeks of incubation due to the slow growth rate of the Mycobacterium tuberculosis complex. The radiometric BACTEC 460 TB system considerably improves the isolation and decreases the time required to detect mycobacterium. However, this procedure is still labor-intensive and requires attention to special safety and regulatory issues due to radioisotopes. BACTEC 460 is used in many laboratories worldwide but the increasing cost of radioactive waste disposal promoted the manufacturer to develop alternative systems. Contamination is controlled by the addition of a mixture of polymyxin $\mathrm{B}$, amphotericin $\mathrm{B}$, nalidixic acid, trimethoprim and azlocillin (PANTA) reconstituted with a polyoxyethylene solution. The BACTEC $460 \mathrm{~TB}$ is comparatively low in nutrient content and only detects organisms that metabolize the 14C labeled substrate Palmitic acid. Many contaminants do not metabolize this substrate [26].

BACTEC MGIT 960: BACTEC mycobacterial growth indicator tube (MGIT) $960 \mathrm{~TB}$ system is an automated liquid in-vitro diagnostic instrument designed for the rapid detection of mycobacterium from specimens. The system has a 960-tube capacity useful in dealing with large specimen loads. For drug susceptibility test using BACTEC MGIT 960, drug-containing and drug-free control vials are inoculated with standardized inoculums of the $M$. tuberculosis isolate and entered into the machine in a special rack-carrier with a printed barcode; it is read by the machine when entering the tubes to identify the test and apply adequate algorithm for susceptibility interpretation. Because of 
higher contamination, MGIT 960 requires careful processing and handling of specimens $[27,28]$.

The MGIT system contains $4 \mathrm{ml}$ of an enriched Middle brook 7H9 broth with $0.25 \%$ glycerol and added oleic acid, albumin, dextrose, and catalase (OADC) and PANTA antibiotics to reduce contamination. A fluorescent indicator quenched by oxygen is embedded in silicone on the bottom of a round-bottom tube. When the growing mycobacterium is consuming oxygen, the fluorescence can easily be detected visually with the aid of 365 -nm UV light. The fluorescent compound is sensitive to the presence of oxygen dissolved in the broth. The initial concentration of dissolved oxygen quenches the emission from the compound, and little fluorescence can be detected. Finally, actively growing and respiring microorganisms consume the oxygen, which permits the compound to fluoresce [29].

BacT/ALERT 3D system: Standard aerobic media are widely used for culturing blood with the BacT/ALERT and BACTEC9240 automated continuously monitoring instrument systems. Although similarly composed of soybean-casein digest broths, the formulations of the standard aerobic media available for these instruments differ from each other in supplements and in sodium polyanetholesulfonate concentration. Blood samples from patients with suspected bloodstream infection were inoculated at into non-vented BM and BD standard aerobic blood culture bottles and incubated in their respective instruments. Ten millilitres of blood was placed in random order into each of three blood culture bottles: a BM standard aerobic bottle (BacT/AlertSA), a BD standard aerobic bottle (BACTEC Standard 10 Aerobic/F) and a BM anaerobic FAN bottle [30].

\section{BD BACTEC ${ }^{\mathrm{rm}}$ FX fluorescent techniques}

The BACTEC $^{\mathrm{ma}}$ FX and the BACTEC ${ }^{\mathrm{m} x} 9000$ series of blood culturing instruments offer performance safety, reliability, ease of use and quality service. The BACTEC FX System builds on previous BACTEC instrumentation; it goes much further with exciting innovations such as: vial-activated workflow, advanced ergonomics, remote alarms and blood culture observation and customer-focused data management. The instrument design provides optimal, easy-to-use workflow in a compact, modular design [31]. It uses infrared spectrophotometers to monitor carbon dioxide produced by microorganisms on a continuous basis. BD BACTEC Plus media on the new BACTEC FX and the incumbent 9000 series blood culture systems utilize cationic-exchange and adsorbent nonionic resins to remove antibiotics from blood samples, and can detect slow growing organisms such as Hemophilus and Neisseria [32]. When microorganisms are present in culture vials, they metabolize nutrients in the culture medium, releasing carbon dioxide into the medium. A dye in the sensor at the bottom of the vial reacts with $\mathrm{CO}_{2}$. This modulates the amount of light that is absorbed by a fluorescent material in the sensor. A photo detector at each station measures the level of fluorescence, which corresponds to the amount of $\mathrm{CO}_{2}$ released by organisms. Then the measurement is interpreted by the system according to pre-programmed positivity parameters [24,33].

BACTEC 9120: It is an automated blood culture system involving 120 test bottles with a complete set of Plus Aerobic/F and Plus Anaerobic/F bottles. Although very few bloodstream infections (1.8\%) were associated with obligate anaerobes, the traditional routine use of anaerobic bottles was confirmed because of their usefulness, not only in the detection of anaerobes, but also in that of gram-positive cocci and fermentative gram-negative bacilli. These systems are extensively used in order to increase the rapidity of isolation and identification of microorganisms in blood samples, and paired aerobic and anaerobic blood culture bottles are commonly used to obtain better overall recovery of microorganisms. With 8 to $10 \mathrm{ml}$ each of fresh, whole blood were collected and placed as soon as possible in a BACTEC 9120 automated blood culture system. Specimens were incubated at $35^{\circ} \mathrm{C}$ with continuous agitation, and a fluorescence technology was used to evaluate the quantity and rate of $\mathrm{CO}_{2}$ production, as these are indicative of microbial growth. The protocol duration of incubation was 120 hrs for Plus Aerobic/F and Plus Anaerobic/F bottles [34].

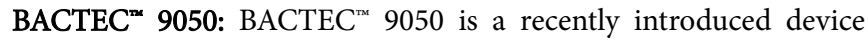
with a capacity of 50 bottles, a small, self-contained, automated system designed for processing three to five blood cultures per day. In addition to the difference in capacity, BACTEC 9050 differ from the larger systems by agitating the bottles continuously and rotating the bottles to be read by one of three detectors. The computer used to monitor the BACTEC 9050 bottles in contained within the system. The culture bottles were arranged in 3 concentric rings and incubated at $35^{\circ} \mathrm{C}$ and continuously agitated for maximum recovery of organisms. Positive cultures were flagged by an indicator light on the front of the instrument, an audible alarm and were displayed on the LCD screen. The cultures were centrifuged in $\mathrm{BD}$ vacutainer at $3000 \mathrm{rpm} / 20$ minutes. Smears from the deposit were stained with Gram stain and subculture onto sheep blood agar plates susceptibility testing was done using automated Siemens Micro scan Walkway system with synergy panels. Several media are available for use with the BACTEC ${ }^{\text {m }}$ FX system. These include: BACTEC ${ }^{\mathrm{rm}}$ Standard/10 Aerobic/F, BACTEC ${ }^{\mathrm{m}}$ Plus Aerobic /F, BACTEC ${ }^{\mathrm{m}}$ Standard Anaerobic /F, BACTEC ${ }^{\mathrm{m} x}$ Peds Plus and BACTEC ${ }^{\mathrm{rx}}$ Lytic/10 Anaerobic /F [25].

\section{The VITEK system}

The VITEK was the first automated instrument developed in the 1960s that allowed the direct inoculation of positive blood cultures into the identification cards. It uses small plastic cards containing multiple wells filled with either biochemical substrates or antibiotic dilutions. Cards can be used for both identification and susceptibility testing for most aerobic gram-positive and gram-negative organisms. The system uses a manual filler sealer to inoculate the cards with the organism. After inoculation, a reader incubator module uses a single wavelength of light to measure turbidity and color changes in the card wells. Robotics technology is used to move the cards every 15 minutes to different reading areas. The VITEK then uses regression analysis to calculate the MIC [35,36]. The system is available in three formats (VITEK 2 compact, VITEK 2 and VITEK $2 \mathrm{XL}$ ) that differ in increasing levels of capacity and automation. All three systems accommodate the same colorimetric reagent cards that are incubated and interpreted automatically [36].

VITEK 2: The VITEK 2 system is the second generation of VITEK and gives a more sophisticated model of data analysis as well as a fully automated process for card identification, organism suspension dilution, and card filling. The VITEK 2 system detects metabolic changes by fluorescence-based techniques which facilitate the identification of gram-negative bacteria within $3 \mathrm{hrs}$. Once these steps are complete, the VITEK 2 seals the cards into a chamber to prevent contamination during processing. The cards are then loaded into the reader incubator, which ejects them at the end of testing. The VITEK 2 uses colorimetric technology with three wavelengths of light to provide broad profiles for the most clinically significant organisms. The analysis is performed by using algorithms to look at a variety of parameters and test conditions to ensure accurate results and early 
detection of resistance mechanisms through proprietary software. It monitors kinetics of bacterial growth and calculates MICs using a unique algorithm $[13,36]$.

Identification with VITEK 2 system is performed with ID-GNB cards. The sixty four well plastic ID-GNB cards contain 41 biochemical tests, including 18 tests for sugar assimilation, 18 tests for sugar fermentation, 2 decarboxylase tests and 3 miscellaneous tests (for urease, utilization of malonate, and tryptophane deaminase). With a vacuum device, the cards are inoculated with a 0.5 McFarland suspension of the organism prepared from 18-20 hrs old Columbia sheep blood agar plate and then automatically sealed and manually inserted inside the VITEK 2 reader-inoculators module. Fluorescence is measured every $15 \mathrm{~min}$ and the results of identification are determined after 3 hrs $[13,36]$.

The reagent cards have sixty four wells that can each contain an individual test substrate. Substrates measure various metabolic activities such as acidification, alkalinization, enzyme hydrolysis and growth in the presence of inhibitory substances. An optically clear film present on both sides of the card allows for the appropriate level of oxygen transmission while maintaining a sealed vessel that prevents contact with organism-substrate mixtures. There are currently four reagent cards available for the identification of various organism classes as follows: GN-Gram-negative fermenting and non-fermenting bacilli, GP-Gram-positive cocci and non-spore-forming bacilli, YSTyeasts and yeast-like organisms and BCL-Gram-positive spore-forming bacilli [36].

VITEK 2 Compact: This format focuses on the industrial microbiology-testing area while also having application for low to middle volume clinical laboratories. Features developed for industrial microbiology include $21 \mathrm{CFR}$ compliance and a colorimetric reagent card used to identify the spore-forming Gram-positive bacilli (i.e., Bacillus and related genera). The other colorimetric reagent cards (GN, GP) apply to all system formats for both industrial and clinical laboratories [37].

\section{The versa TREK system}

The Versa TREK system is adapted to accommodate small or big volumes of blood culture bottles and with its 96 bottle capacity is special for smaller laboratories. This system also has the advantage of using small volumes of blood from as little as $0.1 \mathrm{ml}$ to $10 \mathrm{ml}$. The 80 $\mathrm{ml}$ broth creates an optimal blood-to-broth ratio of 1:9 to minimize, by dilution, the effect of host serum factors and antimicrobials. Aerobic bottles are vortexed with a stir bar to enhance oxygenation of broth. The Versa TREK system is based on monitoring pressure changes in the bottle headspace due to bacterial metabolism and these changes are measured every 24 minutes based on Versa TREK instrument specifications. This system is neither limited by organisms that produce low $\mathrm{CO}_{2}$ concentrations as it detects any gas produced or consumed by microorganisms nor by a high amount of white blood cells [38].

Versa TREK is a fully automatic blood culture diagnostic instrument that detects the change in the gas pressure in the bottle head space. The gas consumption and production of the bacteria are reinforced by high nutrition media. There are only two bottles that are aerobic and anaerobic to be able to detect all types of bacteria. Versa TREK microbial detection media is based on an enhanced media formulation that delivers unparalleled versatility, broad range recoverability, unique response growth curve, vortexing agitation, unquestioned reliability and simple ease of use. A special bottle media do not need any special additive at all. In addition, the tornado stir method that is a new technology is built in an aerobic bottle so that oxygen may always become abundant while it cultures [38,39].

\section{Blood Culture Techniques in Ethiopian}

In Ethiopia, blood culture techniques are rarely used, but few reports from Jimma University Hospital [40], Gondar University Hospital [41] and Tikur Anbessa Specialized Hospital [42], showed blood culture isolates from data about the role and frequency of invasive bacteria on febrile adult cases and susceptibility patterns of these bacteria. These confirmed that blood culture techniques provide basic information in identification of bacteria and empirical choice of antibiotics for treatment of bacteremia [43]. BACTEC system and MGIT systems are available in Ethiopian Public Health Institute (EPHI) for TB diagnosis, and API including BACTEC also found in regional and specialized private laboratories such as international clinical laboratory (ICL). A prospective cross-sectional study at Jimma University Specialized Hospital among 260 febrile cases of blood culture, a total of 23 bacterial strains were isolated, with a culture positivity rate of $8.8 \%$. The overall rate of isolation were $60.9 \%(n=14)$ for gram positive bacteria and $39.1 \%(n=9)$ for gram negative bacteria. The predominant isolates were coagulase negative staphylococci 6 (26.1\%) followed by S. aureus $5(21.7 \%)$ and the least were Salmonella species $1(4.3 \%)$ [40].

Another study done among 181 clinically bactereamia suspected cases at Gondar University Specialized Hospital, there were 48 (32.4\%) and $10(30.3 \%)$ culture proven early and late onset neonatal sepsis cases respectively. The predominant bacterial groups were gram negatives, $36(62.1 \%)$, as compared to gram positives. More than $70 \%$ of Staphylococcus aureus and 73\% of Klebsiella ozaenae were isolated from neonates delivered in the hospital. Forty four percent (44\%) of gram negatives were resistant to third generation cephalosporins and $41.2 \%$ of isolated Staphylococcus aureus were found to be methicillin resistant [41]. In Tikur Anbessa Specialized Hospital, from total of 1,225 children visiting the outpatient paediatric departments with fever and diarrhea, 65 isolates of Salmonella and 48 Salmonella strains were found in blood culture [42].

\section{Conclusions and Recommendations}

Blood culture systems and practices that maximize the recovery of pathogens are imperative in the investigation of septicemic patients to ensure the timely delivery of appropriate antimicrobials. The detection and identification of bacteria from the blood of patients is one of the most important roles of the clinical microbiology laboratory. The automated methods like BACTEC and VITAK 2 generally give better result, reduced error and minimum turnaround time. They provide rapid result and help in the reduction of disease mortality, morbidity and reduction of drug resistance bacterial strain development. In our country Ethiopia, these automated machines rarely found. The BACTEC system, MGIT and API are found in EPHI and in some regional and specialized private laboratories.

Generally, from our article review on emerging blood culture techniques, these techniques are rarely known and used. We thereby would like to recommend some points to laboratory professionals, the concerned bodies and other stakeholders to apply and practice these sophisticated technologies in our country to improve the laboratory service in identifying and testing susceptibility to drugs for various microorganisms. The government also should allocate appropriate 
budget to laboratory service provider institutions for machines, reagents and maintenance equally with other sectors to function properly. The short turnaround times of these techniques are very important for prompt, appropriate treatment of bloodstream infections. Therefore, investing on these techniques is unquestionable for better vision and to build healthy community. Finally, it is better we professionals focus both in theoretical and practical areas of these emerging blood culture techniques as well as teaching schools.

\section{Conflict of Interest}

The authors declare that there is no conflict of interest regarding the publication of this review.

\section{References}

1. Tissari P, Zumla A, Tarkka E, Mero S, Savolainen L, et al. (2010) Accurate and rapid identification of bacterial species from positive blood cultures with DNA-based microarray platform: an observational study. Lancet 375: 224-230.

2. Fenollar F, Raoult D (2007) Molecular diagnosis of bloodstream infections caused by non-cultivable bacteria. Int J Antimicrob Agents 30: S7-15.

3. Kok J, Thomas LC, Olma T, Chen SA, Iredell JR (2011) Identification of Bacteria in Blood Culture Broths Using Matrix-Assisted Laser Desorption-Ionization Sepsityper TM and Time of Flight Mass Spectrometry. Plos One 6: 8.

4. Karunakaran R, Raja NS, Quek KF, Hoe VW, Navaratnam P (2007) Evaluation of the routine use of the anaerobic bottle when using the BACTEC blood culture system. J Microbiol Immunol Infect 40: 445-449.

5. Jansen GJ, Mooibroek M, Idema J, Harmsen HJ, Welling GW, et al. (2000) Rapid Identification of Bacteria in Blood Cultures by Using Fluorescently Labeled Oligonucleotide Probes. J Clin Microbiol 38: 814-817.

6. Ng SY, Kwang LL, Tan TY (2007) Identification of Gram-negative bacilli directly from positive blood culture vials. J Med Microbiol 56: 475-479.

7. Kireçci E, Ozkanlar Y, Aktas MS, Uyanik MH, Yazgi H (2010) Isolation of pathogenic aerobic bacteria from the blood of septicaemic neonatal calves and the susceptibility of isolates to various antibiotics. J S Afr vet Ver 81:110-113.

8. Paolucci M, Landini MP, Sambri V (2010) Conventional and molecular techniques for the early diagnosis of bacteraemia. Int J Antimicrob Agents 36: S6-16.

9. Buttery JP (2002) Blood cultures in newborns and children: Optimizing an everyday test. Arch Dis Child Fetal Neonatal Ed 87:25-28.

10. Weinstein MP (2003) Blood Culture Contamination: Persisting Problems and Partial Progress. J. Clin. Microbiol 41:2275-2278.

11. Stager CE, Davis JR (1992) Automated Systems for Identification of Microorganisms. Clinical microbiology reviews 5: 302-327.

12. Shetty N, Hill G, Ridgway GL (1998) The VITEK analyzer for routine bacterial identification and susceptibility testing: protocols, problems, and pitfalls. J Clin Pathol 51:316-323.

13. O'Hara CM (2005) Manual and Automated Instrumentation for Identification of Enterobacteriaceae and Other Aerobic Gram-Negative Bacilli. Clin Microbiol Rev 18:147-162.

14. Tang YW, Stratton CW (2006) Advanced Techniques in Diagnostic Microbiology. Springer Science and Business Media, LLC 63-71.

15. Holmes B, Costas M, Thaker T, Stevens M (1994) Evaluation of two BBL Crystal systems for identification of some clinically important gramnegative bacteria. J Clin Microbiol 32:2221-2226.

16. Wilmot BA, Chu MC (1996) Identification of Yersinia pestis by BBL Crystal Enteric/Nonfermenter Identification System. J Clin Microbiol 34: 2829-2830.

17. Cavallaro JJ, Wiggs LS, Miller JM (1997) Evaluation of the BBL Crystal Anaerobe identification system. J Clin Microbiol 35:3186-3191.
18. Rennie RP, Brosnikoff C, Shokoples S, Reller LB, Mirrett S, et al. (2008) Multicenter Evaluation of the New Vitek 2 Neisseria-Haemophilus Identification Card. Journal of clinical microbiology 46:2681-2685.

19. Washington JA II, Yu PKW, Martin WJ (1971) Evaluation of accuracy of multi-test micro method system for identification of Enterobacteriaceae. Applied Microbiology 22: 267-269.

20. Popovic NT, Rakovac RC, Perovic IS (2007) Commercial phenotypic tests (API 20E) in diagnosis of fish bacteria: a review. Veterinarni Medicina 52:49-53.

21. Janda WM, Ristow K, Novak D (1994) Evaluation of RapiDEC Staph for identification of Staphylococcus aureus, Staphylococcus epidermidis and Staphylococcus saprophyticus. J Clin Microbiol 32:2056-2059.

22. Griethuysen A, Buiting A, Goessens W, Peter van Keulen, Rob Wintermans, et al. (1998) Multicenter Evaluation of a Modified Protocol for the RAPIDEC Staph System for Direct Identification of Staphylococcus aureus in Blood Cultures. J Clin Microbiol 36: 3707-3709.

23. Poutrel B, Ryniewicz HZ (1984) Evaluation of the API 20 Strep system for species identification of streptococci isolated from bovine mastitis. J Clin Microbiol 19: 213-214.

24. Gopi A, Ravikumar KL Ambarish MG, Shwethalatha NN, Shree SK, et al. (2011) Time to Positivity of Microorganisms with BACTEC 9050:- An 18month Study among Children of 28 Days to 60 Months in a South Indian Tertiary. Intl J Microbiol Res 2: 12-17.

25. Fuller DA (2008) BD BACTEC ${ }^{\mathrm{m}}$ FX First Experiences. Fall 19:01-16.

26. Rodrigues C, Shenai S, Sadani M, Sukhadia N, Jani M, et al. (2009) Evaluation of the BACTEC MGIT 960 TB System for Recovery and identification of Mycobacterium Tuberculosis Complex in a high through Put Tertiary Care Centre. Indian J Med Microbiol 27:217-221.

27. Kadam M, Govekar A, Shenai S, Sadani M, Salvi A, et al. (2010) Can Cord Formation in BACTEC MGIT 960 Medium be used as a Presumptive Method for Identification of M. Tuberculosis Complex? Indian J Tuberc 57:75-79.

28. Safwat TM, Elmasry AA, Salem HM (2012) BACTEC MGIT 960 System in the Susceptibility Testing of Mycobacterium Tuberculosis to the Four First-Line Antituberculous Drugs. Comparison with Lowenstein Jensen Media. Egypt J Bronchol 6:1.

29. Sharma B, Pal N, Malhotra B, Vyas L, Rishi S (2010) Comparison of MGIT 960 and pyrazinamidase activity assay for pyrazinamide susceptibility testing of Mycobacterium tuberculosis. Indian J Med Res 132:72-76.

30. Mirrett S, Reller LB, Petti CA, Woods CW, Vazirani B, et al. (2003) Controlled Clinical Comparison of BacT/ALERT Standard Aerobic Medium with BACTEC Standard Aerobic Medium for Culturing Blood. J Clin Microbiol 41:2391-2394.

31. BBL $^{\mathrm{Tm}}$ Crystal $^{\mathrm{Ts}}$ Enteric/Nonfermenter ID Kit. BD BACTEC ${ }^{\mathrm{mm}}$ Instrumented Blood Culture Systems. Accessed on 28Apr 2016.

32. Miller NS, Rogan D, Orr BL, Whitney D (2011) Comparison of BD BACTEC Plus Blood Culture Media to Versa TREK Redox Blood Culture Media for Detection of Bacterial Pathogens in Simulated Adult Blood Cultures Containing Therapeutic Concentrations of Antibiotics. J Clin Microbiol 49:1624-1627.

33. White Papers BD BACTEC ${ }^{\mathrm{m}} \mathrm{FX}$ bacterial identification instrument system. Accessed on 28Apr 2016.

34. Chiarini A, Palmeri A, Amato T, Immordino R, Giammanco A, et al. (2008) Detection of Bacterial and Yeast Species with the BACTEC 9120 Automated System with Routine Use of Aerobic, Anaerobic and Fungal Media. Journal of Clinical Microbiology 46: 4029-4033.

35. Joyances P, Conejos MC, Martinez LM, Perea EJ (2001) Evaluation of the VITEK 2 System for the Identification and Susceptibility Testing of Three Species of Non-fermenting Gram-Negative Rods Frequently Isolated from Clinical Samples. J Clin Microbiol 39: 3247-3253.

36. Kipper KM, Boles DM, Mohr JF, Wagner A (2009) Antimicrobial Susceptibility Testing: A Primer for Clinicians. Pharmacotherapy 29:1326-1343. 
Citation: Dilnessa T, Demeke G, Mengistu G, Bitew A (2016) Emerging Blood Culture Technologies for Isolation of Blood Pathogens at Clinical Microbiology Laboratories. J Med Microb Diagn 5: 227. doi:10.4172/2161-0703.1000227

Page 7 of 7

37. Pincus DH. Microbial Identification Using the biomérieux VITEK 2 System.

38. Dreyer AW, Ismail NA, Nkosi D, Kathy Lindeque, Marliza Matthews, et al. (2011) Comparison of the Versa TREK blood culture system against the Bactec 9240 system in patients with suspected bloodstream infections. Ann Clin Microbiol Antimicrob 10: 4.

39. Versa TREK fully-automatic blood culture diagnostic device. Thermo Scientific. Assessed on 28-Apr 2016.

40. Zenebe T, Kannan S, Yilma D, Beyene G (2011) Invasive Bacterial Pathogens and their Antibiotic Susceptibility Patterns in Jimma University Specialized Hospital, Jimma, Southwest Ethiopia. Ethiop J Health Sci 21:1-8.
41. Gebrehiwot A, Lakew W, Moges F, Beyene Moges, Belay Anagaw, et al. (2012) Predictors of positive blood culture and death among neonates with suspected neonatal sepsis in Gondar University Hospital, Northwest Ethiopia. Euro J Exp Bio 2: 2212-2218.

42. Beyene G, Nair S, Asrat D, Mengistu Y, Engers H, et al. (2011) Multidrug resistant Salmonella Concord is a major cause of Salmonellosis in children in Ethiopia. J Infect Dev Ctries 5: 23-33.

43. (2009) Clinical and Laboratory Standards Institute. Performance standards for antimicrobial disk susceptibility tests; approved standardtenth edition M02-A10. National Committee for Clinical Laboratory Standards 29. 